\title{
Effects of oxygen adsorption on carbon nanotube field emitters
}

\author{
Noejung Park, ${ }^{1}$ Seungwu Han, ${ }^{1,2}$ and Jisoon $\mathrm{Ihm}^{1}$ \\ ${ }^{1}$ School of Physics, Seoul National University, Seoul 151-742, Korea \\ ${ }^{2}$ Center for Strongly Correlated Materials Research, Seoul National University, Seoul 151-742, Korea
}

(Received 23 April 2001; published 20 August 2001)

\begin{abstract}
Effects of oxygen adsorption on the field emission of carbon nanotubes are studied through first-principles calculations. Calculated emission currents are significantly enhanced when oxygen is adsorbed at the tip and the underlying physics is explained in terms of the change in the electronic structure by oxidation and the local field increase at the adsorption site. The issue of the current degradation accompanied by the oxidative etching is also addressed. The field-emission-microscopy images on the phosphor screen are simulated, displaying various patterns characteristic of each adsorption configuration.
\end{abstract}

DOI: 10.1103/PhysRevB.64.125401

PACS number(s): 79.70.+q, 61.46.+w, 73.63.Fg, 31.15.Ar

Ever since the discovery of carbon nanotubes (CNT's), ${ }^{1}$ a lot of effort has been given to utilizing their unique electronic and structural properties. Among a wide range of prospective applications, using this material as electron emitters in the field emission display is regarded as a promising area that could be realized in the near future. ${ }^{2}$ Carbon nanotubes are ideal as a field-emitting material because of their high aspect ratios and strong carbon bonding, and nanotube field emitters have been reported to achieve low-threshold voltages, large current densities, and long-term stability in the emission current. $^{3-6}$

The effects of ambient gases on the stability of the emission current are of great interest in the operation of field emitters. Especially, the influence of oxygen on the field emission has been extensively studied because oxygen constitutes a significant portion of the ambient gas and is chemically very reactive. The oxygen adsorbed on micron-sized metal tips is known to reduce the emission current by inducing a surface dipole which increases the work function. ${ }^{7}$ In the case of the nanotube field emission, on the other hand, recent experimental studies have shown that the adsorption of ambient gas such as $\mathrm{H}_{2} \mathrm{O}$ and $\mathrm{O}_{2}$ instantaneously induces a large current increase. 8,9 The currents from the adsorbateinduced states dominate the field emission and remain stable even under a high-vacuum condition. However, a long-term exposure to $\mathrm{O}_{2}$ or $\mathrm{H}_{2} \mathrm{O}$ with a pressure higher than $10^{-7}$ Torr resulted in an irreversible current degradation. ${ }^{6,10}$ These puzzling effects of oxygen on the nanotube field emission have not been clearly understood yet.

In this paper, we study field emission from oxygenadsorbed CNT's through realistic quantum mechanical calculations, focusing on the current change induced by the adsorbed oxygen. We consider two types of adsorption, atomic or molecular, as possible configurations of oxygen on the nanotube tip. We are going to show that the emission currents are always increased with the adsorption of oxygen. We will also suggest that the oxidative etching might play an essential role in the long-term degradation of the emission currents.

The binding energies and the equilibrium adsorption geometries are calculated using the pseudopotential method ${ }^{11}$ within the local density approximation. ${ }^{12}$ The energy cutoff in the plane-wave expansion is set to $65 \mathrm{Ry}$. The system is fully relaxed until the total energy converges within $0.01 \mathrm{eV}$. The emission currents of the relaxed geometry are calculated with an $a b$ initio approach. ${ }^{13}$ In brief, this method is a twostep process. At first, the electronic structure and the potential under an external field are calculated self-consistently using a localized basis. ${ }^{14}$ Next, we switch the basis to plane waves and evaluate the tunneling rates of electrons into the vacuum (on the anode side) by solving time-dependent Schrödinger equations.

In Fig. 1(a), the $(5,5)$ nanotube used for calculating the adsorption geometry and the binding energy is shown. The emission side of the nanotube is closed with a hemisphere of $\mathrm{C}_{60}$, and the other side is passivated with hydrogen atoms. The length of the tube is about $9 \AA$ and the dimension of the supercell is large enough $(12 \AA \times 12 \AA \times 17 \AA)$ to avoid interaction between different supercells. We first investigate the atomic adsorption of oxygen. Because of the high chemical activity of the pentagonal defect, the cap of the nanotube is more reactive to oxygen than the nanotube wall. ${ }^{15}$ Two stable adsorption geometries O/CNT- $p h$ and O/CNT- $h h$ are presented in Figs. 1(b) and 1(c), respectively. In O/CNT-ph, the oxygen atom is located over the pentagon-hexagon $(p-h)$ bond [see Fig. 1(a)] while it is located over the hexagonhexagon $(h-h)$ bond [see Fig. $1(\mathrm{a})]$ in O/CNT- $h h$. The bind-

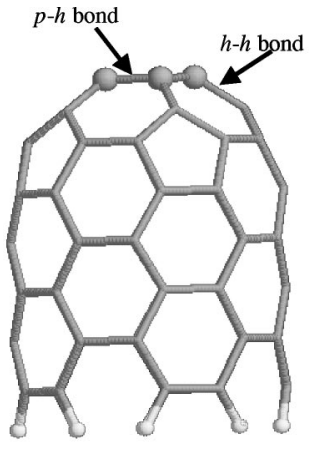

(a)

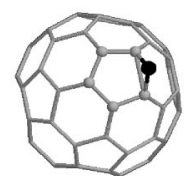

(b)

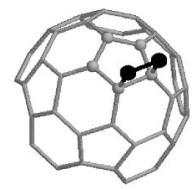

(d)

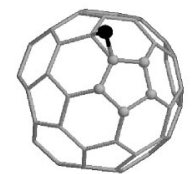

(c)

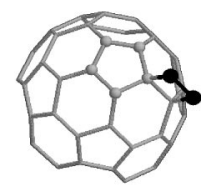

(e)
FIG. 1. Oxygen adsorption configurations on the $(5,5)$ carbon nanotube cap: (a) is the side view of the bare cap without oxygen. (b) O/CNT- $p h$, (c) O/CNT- $h h$, (d) $\mathrm{O}_{2} / \mathrm{CNT}-p h$, and (e) $\mathrm{O}_{2} / \mathrm{CNT}-h h$ geometries (see text for abbreviations). In (b)-(e), only the cap and adsorbates are shown for visual clarity. 
TABLE I. The emission current $(I)$ and the binding energy $\left(E_{b}\right)$ for the model configurations. $E_{b}=$ $-E\left(\mathrm{O}_{x} / \mathrm{CNT}\right)+E(\mathrm{CNT})+E\left(\mathrm{O}_{x}\right)$, where $x$ is 1 or 2 .

\begin{tabular}{cccc}
\hline \hline Adsorption geometry & $I\left(\mu \mathrm{A}, E_{\text {ext }}=0.7 \mathrm{~V} / \AA\right)$ & $I\left(\mu \mathrm{A}, E_{\text {ext }}=0.5 \mathrm{~V} / \AA\right)$ & $E_{b}(\mathrm{eV})$ \\
\hline O/CNT- $p h$ & 1.912 & 0.058 & 6.73 \\
O/CNT- $h h$ & 0.652 & 0.020 & 6.60 \\
$\mathrm{O}_{2} /$ CNT- $p h$ & 5.611 & 0.214 & 1.66 \\
$\mathrm{O}_{2} /$ CNT- $h h$ & 3.016 & 0.122 & 3.02 \\
CNT & 0.276 & 0.004 & \\
\hline \hline
\end{tabular}

ing energy of the oxygen atom for each configuration is presented in Table I. Even though the difference in the binding energy between $\mathrm{O} / \mathrm{CNT}-p h$ and $\mathrm{O} / \mathrm{CNT}-h h$ is rather small $(0.13 \mathrm{eV})$, their equilibrium geometries and local electronic structures are quite different from each other. In the O/CNT-ph case, two carbon atoms bonded to the oxygen atom extrude out of the cap and the distance between them is increased to $1.91 \AA$ compared with other $p$ - $h$ bond lengths of $1.45 \AA$. In contrast, the structural deformation of the cap is negligible in the O/CNT- $h h$ geometry. The difference in the local electronic structures of two adsorption geometries will be discussed later in relation to emission currents. Our results agree well with those of the Hartree-Fock calculation on $\mathrm{C}_{60} \mathrm{O},{ }^{16}$ as expected from the aforementioned geometrical identity between the hemisphere of $\mathrm{C}_{60}$ and the cap of the $(5,5)$ nanotube.

We have found two stable adsorption geometries for an $\mathrm{O}_{2}$ molecule at the cap as shown in Figs. 1(d) and 1(e), and they are named $\mathrm{O}_{2} / \mathrm{CNT}-p h$ and $\mathrm{O}_{2} / \mathrm{CNT}-h h$, respectively. The binding energy of $\mathrm{O}_{2}$ at the top of the $p$ - $h$ bond in the $\mathrm{O}_{2} / \mathrm{CNT}-p h$ geometry is $1.66 \mathrm{eV}$ (see Table I), compared with $3.02 \mathrm{eV}$ for the $\mathrm{O}_{2} / \mathrm{CNT}-h h$ geometry. The difference $(1.36 \mathrm{eV})$ is larger than that in the $\mathrm{O}_{2}$ adsorption on $\mathrm{C}_{60}(0.82$ $\mathrm{eV})$ reported in Ref. 17. These values are smaller than the binding energy of $\mathrm{O}_{2}$ on the open edge of the CNT $(4.61 \mathrm{eV})$ reported in Ref. 17, but much larger than that on the wall of the CNT $(0.25 \mathrm{eV}) .{ }^{18}$ We deduce that a greater curvature (i.e., a smaller radius of curvature) causes a stronger $\sigma-\pi$ bond mixing and a stronger binding of foreign molecules. Since commonly synthesized CNT's in an actual experiment would have a larger cap (i.e., a smaller curvature) than our model system, the binding energy of $\mathrm{O}_{2}$ on the cap is probably smaller than those reported here but still larger than that on the wall of the CNT $(0.25 \mathrm{eV})$, and we expect a substantial oxygen binding on a larger cap of the CNT.

Now we present results for the field emission current of each adsorption geometry. We consider much longer nanotubes $(\sim 30 \AA)$ here to investigate the properties of the tube end more realistically. As described before, a localized basis set is used in calculating the self-consistent potentials and the eigenstates of these longer nanotubes. Pseudoatomic orbitals $^{19}$ are used and four orbitals (one for $s$ and three for $p$ ) are employed for each atom. ${ }^{20}$ The vacuum in the axial $(z)$ direction is $30 \AA$ long and the distance between neighboring nanotubes in the $x y$ direction is $13 \AA$. The emission current of each state is evaluated from the decrease rate of the charge remaining inside the tube in the time interval between 30 a.u. and 40 a.u. ( 1 a.u. $=0.0242 \mathrm{fs}$ ) after the initiation of time evolution. ${ }^{13}$ In Table I, the calculated emission currents of the model systems are presented for the external electric fields $\left(E_{\text {ext }}\right)$ of $0.7 \mathrm{~V} / \AA$ and $0.5 \mathrm{~V} / \AA$. It is noticeable that the oxygen adsorption significantly enhances the emission current in all cases and the amount of the current increase is dependent on the detailed binding configurations. Especially, the emission currents change by several orders of magnitude when oxygen binds as a molecule. When $E_{\text {ext }}$ is $0.5 \mathrm{~V} / \AA$, for example, the emission current jumps from $\sim 1$ to $\sim 100 \mathrm{nA}$ upon the molecular adsorption of oxygen, and this amount of current change can actually be found in the experimental results of Ref. 9.

In order to understand the origin of the current enhancement by oxygen adsorption, we inspect the local density of states (LDOS) near the Fermi level for the oxygen and five topmost carbon atoms as presented in Fig. 2. For the cap without oxygen adsorption, doubly degenerate localized

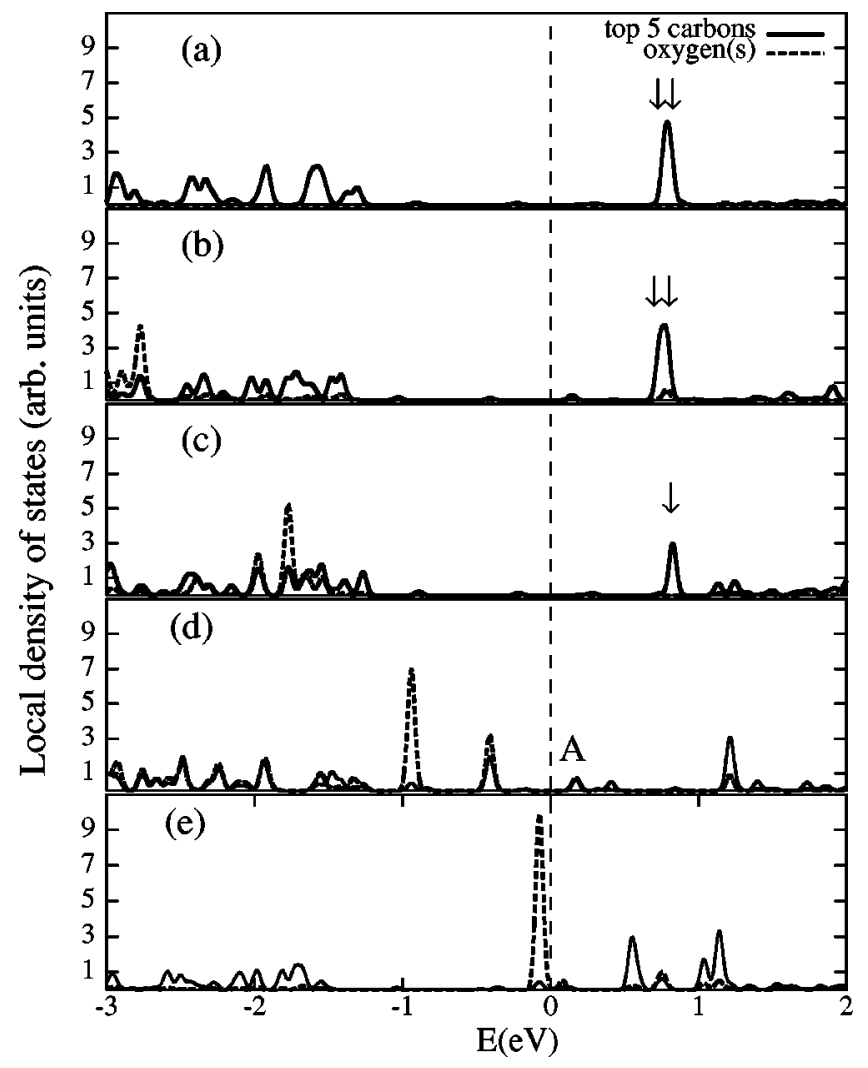

FIG. 2. LDOS for the oxygen and five topmost carbon atoms in each model system. The arrows in (a)-(c) indicate localized states (the double arrow represents a degeneracy). 
states are located at $0.8 \mathrm{eV}$ above the Fermi level as shown in Fig. 2(a). ${ }^{21,22}$ Under external fields, these states become gradually occupied and give dominant contributions $(\sim 70 \%)$ to the emission current. ${ }^{13}$ The $C_{5 v}$ symmetry in this bare cap renders the localized states either symmetric or antisymmetric under a mirror reflection. ${ }^{23}$ In the O/CNT- $p h$ configuration where the oxygen atom is located over the $p-h$ bond, the coupling between the oxygen $p$ states and the localized states of the nanotube is very weak due to different symmetries or too small overlaps. Therefore, the LDOS remains almost unchanged except for a strong oxygen peak at $-2.8 \mathrm{eV}$ in Fig. 2(b) which corresponds to the oxygen $p$ state lying perpendicular to the plane including C-O-C atoms. When the oxygen atom is over the $h-h$ bond in the $\mathrm{O} / \mathrm{CNT}-h h$ geometry, however, the oxygen $p$ state is strongly coupled to one of the localized states which is symmetric under the mirror reflection and the resulting states are located around $2 \mathrm{eV}$ below the Fermi level [Fig. 2(c)]; thus a dominant field-emitting state disappears from the relevant energy range. This explains the large difference in the emission current between O/CNT-ph and O/CNT- $h h$ geometries in spite of the structural similarity. The above characteristics in the LDOS of O/CNT are consistent with the computational results on $\mathrm{C}_{60} \mathrm{O}$ (Ref. 16) where an oxygen atom is found to disrupt the $\pi$ system of $\mathrm{C}_{60}$ only when it is on the $h-h$ bond. It is interesting that the emission current of the O/CNT- $h h$ geometry is still several times greater than that of the bare nanotube even though the number of localized states contributing to the current is only a half (see Table I). We find that this is due to the enhancement of the local electric field accompanied by oxygen adsorption: The local electric field at the position of the oxygen atom of the O/CNT- $h h$ geometry is greater than that at the cap of the bare nanotube by $0.2 \mathrm{~V} / \AA$ when the external field is $0.7 \mathrm{~V} / \AA$. The increase in the local electric field reduces the tunneling distance of electrons, thereby enhancing the emission rates.

The LDOS of $\mathrm{O}_{2} / \mathrm{CNT}-p h$ and that of $\mathrm{O}_{2} / \mathrm{CNT}-h h$ geometries are presented in Figs. 2(d) and 2(e), respectively. The LDOS for the molecular adsorption is characteristically different from that of the atomic adsorption; doubly degenerate $p p \pi^{*}$ states of the $\mathrm{O}_{2}$ molecule remain more or less intact in $\mathrm{O}_{2} / \mathrm{CNT}$, developing large increases in the LDOS around the Fermi level. In the case of the $\mathrm{O}_{2} / \mathrm{CNT}-p h$ geometry, all of the oxygen peaks except peak $A$ in Fig. 2(d) are derived from the $p p \pi^{*}$ states of the $\mathrm{O}_{2}$ molecule. Peak $A$ is generated through a coupling between $p p \pi$ states of $\mathrm{O}_{2}$ and an extended $\pi$ state in the nanotube. Even though this state is more spread out over the nanotube than the localized states, it is most efficient in the field emission of the $\mathrm{O}_{2}$ /CNT- $p h$ geometry, covering $75 \%$ of the total current. The wave function of this state does not have a node in the $x y$ plane and the tunneling barrier to the vacuum is smaller than those of other states, leading to a much enhanced current. For the $\mathrm{O}_{2}$ adsorption at the top of the $h$ - $h$ bond, on the other hand, the strong oxygen peak just below the Fermi level in Fig. 2(e) is mostly of $p p \pi^{*}$ character of the $\mathrm{O}_{2}$ molecule and $80 \%$ of the emission current comes from this state. In contrast to the O/CNT cases, we find that the localized states at the cap in $\mathrm{O}_{2} / \mathrm{CNT}$ are coupled with the oxy-

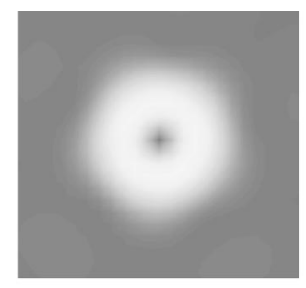

(a)

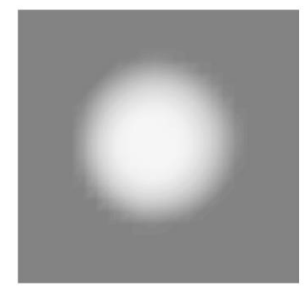

(c)

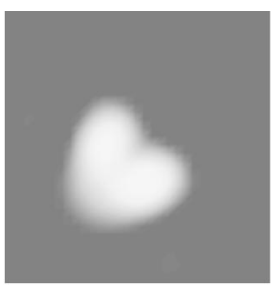

(b)

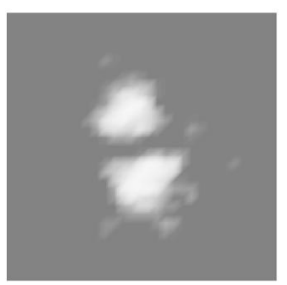

(d)
FIG. 3. Simulated FEM images of the model systems: (a) bare cap or O/CNT-ph, (b) O/CNT- $h h$, (c) $\mathrm{O}_{2} / \mathrm{CNT}-p h$, and (d) $\mathrm{O}_{2} / \mathrm{CNT}-h h$.

gen states and their contributions to the emission current are rather small.

In the results presented so far, the emission current is not reduced upon oxygen adsorption. On the other hand, oxidative etching can take place in a long-term period and cause degradation in the nanotube field emission. In carrying out the theoretical study for this, we are faced with difficulties in finding a model for the complex geometries of the nanotube in the middle of the etching process. ${ }^{24}$ As a representative system of the nanotube during the oxidative etching, we just consider an open $(9,0)$ nanotube with nine oxygen atoms fully covering the open zigzag edge. ${ }^{17}$ It is found that the current of this model system is $65 \%$ of that of the capped $(9,0)$ nanotube without oxygen when $E_{\text {ext }}$ is $0.7 \mathrm{~V} / \AA$. We cannot find any unfavorable factor in the local electric field or in the shape of the emitting state of the oxidized $(9,0)$ nanotube, and only the increase in the work function due to the dipole layer formed by the adsorbed oxygen rim can account for the current reduction. This current can be recovered from degradation by eliminating the oxygen atoms since the open nanotubes with dangling bonds tend to close spontaneously. ${ }^{25}$ However, the oxidative etching in a long time period may cause irreversible structural changes (e.g., shorten the length of the nanotube), which eventually result in an irreversible degradation of the emission currents. Such a sequence of reversible and irreversible degradations in the emission currents caused by oxygen was actually observed in the experiment. ${ }^{6}$

As shown in many experiments, the field emission microscopy (FEM) images on the phosphor screen reveal information on the atomic structure around the tip region. ${ }^{8,26}$ To compare computational results with experiments, we simulate the FEM image of each adsorption geometry as displayed in Fig. 3. The images are obtained by visualizing the square of the electronic wave function at $t=40$ a.u. evaluated on the $x y$ plane located at $10 \AA$ away from the tip. In the case of the bare cap and the O/CNT-ph geometry, a ring 
pattern appears as the contributions of two localized states are added [Fig. 3(a)]. One of the localized states does not contribute in the O/CNT- $h h$ geometry, and a two-lobed image is obtained in Fig. 3(b). When $\mathrm{O}_{2}$ is adsorbed, the oxygen states mainly determine the shape of the images; the $p p \pi$ state of the $\mathrm{O}_{2}$ molecule leads to a single spot for the $\mathrm{O}_{2}$ /CNT-ph geometry [Fig. 3(c)] whereas a two-lobed image appears for the $\mathrm{O}_{2} / \mathrm{CNT}-h h$ geometry because of the nodal plane in the $p p \pi^{*}$ state [Fig. 3(d)]. All of these shapes (a ring, spot, and lobes) were observed in the experiments. ${ }^{8,27}$

In summary, we have investigated the effects of oxygen adsorption on the field emission properties of nanotubes. It is found that emission currents are enhanced in all adsorption configurations studied here and especially large current in creases are observed for the molecular adsorption $\left(\mathrm{O}_{2} / \mathrm{CNT}\right)$ cases. The underlying physics for the current increase is different for atomic and molecular adsorption; the enhancement of the local electric field leads to a current increase for the atomic adsorption $(\mathrm{O} / \mathrm{CNT})$ while the generation of new states induced by the $\mathrm{O}_{2}$ molecule explains the large emission current of the $\mathrm{O}_{2} / \mathrm{CNT}$ configurations. By comparing the current of the capped $(9,0)$ nanotube and the open $(9,0)$ nanotube with the oxygen rim, we suggest that the structural change involving the oxidative etching may be the main reason for the current degradation found in experiments.

This work was supported by the BK21 project of the KRF, the Ministry of Information and Communication of Korea, and Samsung Electronics. The computations were carried out on the CrayT3E of the KORDIC Supercomputing Division.

${ }^{1}$ S. Iijima, Nature (London) 354, 56 (1991).

${ }^{2}$ W.B. Choi et al., Appl. Phys. Lett. 75, 3129 (1999); N.S. Lee et al., Jpn. J. Appl. Phys., Part 1 39, 7154 (2000).

${ }^{3}$ Q.H. Wang, T.D. Corrigan, J.Y. Dai, R.P.H. Chang, and A.R. Krauss, Appl. Phys. Lett. 70, 3308 (1997).

${ }^{4}$ W. Zhu, C. Bower, O. Zhou, G. Kochanski, and S. Jin, Appl. Phys. Lett. 75, 873 (1999).

${ }^{5}$ J.M. Bonard, F. Maier, T. Stockli, A. Chatelain, W.A. de Heer, J.P. Salvetat, and L. Forro, Ultramicroscopy 73, 7 (1998).

${ }^{6}$ K.A. Dean and B.R. Chalamala, Appl. Phys. Lett. 75, 3017 (1999).

${ }^{7}$ B.R. Chalamala, R.M. Wallace, and B.E. Gnade, J. Vac. Sci. Technol. B 16, 2859 (1998).

${ }^{8}$ K.A. Dean and B.R. Chalamala, J. Appl. Phys. 85, 3832 (1999).

${ }^{9}$ K.A. Dean and B.R. Chalamala, Appl. Phys. Lett. 76, 375 (2000).

${ }^{10}$ A. Wadhawan, R.E. Stallcup, II, and J.M. Perez, Appl. Phys. Lett. 78, 108 (2001).

${ }^{11}$ J. Ihm, A. Zunger, and M.L. Cohen, J. Phys. C 12, 4409 (1979).

${ }^{12}$ W. Kohn and L.J. Sham, Phys. Rev. 140, A1133 (1965).

${ }^{13} \mathrm{~S}$. Han and J. Ihm (unpublished).

${ }^{14}$ S. Han and J. Ihm, Phys. Rev. B 61, 9986 (2000).

${ }^{15}$ P.J. Britto, K.S.V. Santhanam, A. Rubio, J.A. Alonso, and P.M. Ajayan, Adv. Mater. 11, 154 (1999).

${ }^{16}$ K. Raghavachari, Chem. Phys. Lett. 195, 221 (1992).

${ }^{17}$ X.Y. Zhu, S.M. Lee, Y.H. Lee, and T. Frauenheim, Phys. Rev. Lett. 85, 2757 (2000).

${ }^{18}$ S.H. Jhi, S.G. Louie, and M.L. Cohen, Phys. Rev. Lett. 85, 1710 (2000).

${ }^{19}$ O.F. Sankey and D.J. Niklewski, Phys. Rev. B 40, 3979 (1989).

${ }^{20}$ We have checked the accuracy of the pseudoatomic orbitals for the model systems in Fig. 1 and found that the energy levels agree well with the plane-wave results within $0.1 \mathrm{eV}$.

${ }^{21}$ P. Kim, T.W. Odom, J. Huang, and C.M. Lieber, Phys. Rev. Lett. 82, 1225 (1999).

${ }^{22}$ D.L. Carrol, P. Redlich, P.M. Ajayan, J.C. Charlier, X. Blase, A. De Vita, and R. Car, Phys. Rev. Lett. 73, 2811 (1997).

${ }^{23}$ A. De Vita, J.-Ch. Charlier, X. Blase, and R. Car, Appl. Phys. A: Mater. Sci. Process. 68, 283 (1999).

${ }^{24}$ M.S.C. Mazzoni, H. Chacham, P. Ordejon, D. Sanchez-Portal, J.M. Soler, and E. Artacho, Phys. Rev. B 60, R2208 (1999).

${ }^{25}$ J. Charlier, A. De Vita, X. Blase, and R. Car, Science 275, 646 (1997).

${ }^{26}$ Y. Saito, K. Hata, and T. Murata, Jpn. J. Appl. Phys., Part 2 39, L271 (2000).

${ }^{27}$ J.-M. Bonard, J.-P. Salvetat, T. Stockli, L. Forro, and A. Chatelain, Appl. Phys. A: Mater. Sci. Process. 69, 245 (1999). 